\title{
Selective drug delivery approaches to lesioned brain through blood brain barrier disruption
}

\section{Zahraa S. Al-Ahmady}

To cite this article: Zahraa S. Al-Ahmady (2018): Selective drug delivery approaches to lesioned brain through blood brain barrier disruption, Expert Opinion on Drug Delivery, DOI: 10.1080/17425247.2018.1444601

To link to this article: https://doi.org/10.1080/17425247.2018.1444601

Accepted author version posted online: 21

Feb 2018.

Published online: 01 Mar 2018.

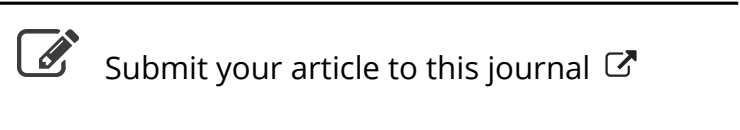

Џ Article views: 3

Q View related articles $₫$

View Crossmark data $\asymp$ 


\title{
Selective drug delivery approaches to lesioned brain through blood brain barrier disruption
}

\author{
Zahraa S. Al-Ahmady
}

Nanomedicine Lab, Division of Pharmacy and Optometry, Faculty of Biology, Medicine and Heath, University of Manchester, UK

\begin{abstract}
Introduction: The development of therapeutics for central nervous system (CNS) disorders is still considered a challenging area in drug development due to insufficient translocation through the blood-brain barrier (BBB). Under normal conditions, BBB restrict the penetration of more than $98 \%$ of blood-borne molecules including drugs to the CNS. However, recent research findings have proven that the nature of the BBB is altered in several neurological conditions. This complexity encourages revisiting drug delivery strategies to the CNS as this can give a wide range of opportunities for CNS drug development.

Areas covered: This review focuses on nanotechnology-based drug delivery platforms designed for selective recruitment into the lesioned brain by taking advantages of BBB disruption that is associated with certain neurological conditions.

Expert opinion: Current CNS therapeutic strategies do not fully address the pathophysiological adaptation of BBB in their design. The lack of selective delivery to the brain lesions has been the culprit behind the failure of many CNS therapeutics. This highlighted the need for smart designs of advanced drug delivery systems that take advantage of BBB structural changes in CNS diseases. Recently, promising examples have been reported in this area, however, more work is still required beyond the preclinical testing.
\end{abstract}

ARTICLE HISTORY

Received 30 July 2017

Accepted 19 February 2018

KEYWORDS

Blood-brain barrier; nanoparticles-based drug delivery; nanomedicine; lesioned brain

\section{Challenges of CNS drug delivery: the BBB obstacle}

The development of therapeutics for central nervous system (CNS) disorders is a significant challenge owing to the privileged protective nature of the brain mediated by the bloodbrain barrier (BBB). Experimental evidence of restrictive movement of solutes from blood to brain tissues dated back to 1880s when Paul Ehrlich first described that many acidic dyes injected into peripheral blood did not stain the CNS [1]. However, it was only in the late 1960s that Reese and Karnovsky found out that the barrier was localized to the endothelium by electron-microscopy studies [2]. Several other studies that followed showed that substances can have variable ratios on both sides of the $\mathrm{BBB}$ and those ratios can be altered in disease situations [3,4]. Thus, BBB emerged as a complex and a dynamic process that controlled the exchange of substances between the blood and CNS. This process was found to be different between substances and was adaptable to pathological conditions [5].

Unlike the microvasculature in the periphery, brain endothelial cells (BECs) are interconnected by tight junctions (TJs) that seal spaces between endothelial cells [6]. TJs form a physical barrier for paracellular transport and limit brain penetration of large hydrophilic macromolecules that would otherwise readily cross the endothelial cells (Figure 1) [7]. Therefore, paracellular route has very limited contribution to the entry of substances into the brain. This is usually for molecules with very long half-lives, small volume of distribution, and very potent CNS effect. Antibodies and erythropoietin (EPO) are examples of that [8].

The lipid nature of BECs allows the entry of small lipophilic molecules with molecular weight $<400 \mathrm{Da}$ by a process known as passive diffusion (Figure 1) [9]. Passive diffusion is nonsaturable process for small lipophilic molecules. The degree by which a substance can undergo passive diffusion also depends on other factors e.g. pharmacokinetic properties, hydrogen bonding, and charge. This process is usually measured by the octanol/water partition coefficient, ideally between 10:1 and 100:1 [9]. Examples of marketed drugs that access the brain by this pathway are steroids and diphenhydramine $[10,11]$.

Active transporters, on the other hand, enable the movement of substances such as essential nutrients, ions, and other endogenous substances into the brain against their concentration gradient in an energy-dependent manner. Many therapeutic molecules utilize this pathway to get access into the CNS such as opioid analgesics, cardiac glycosides, and calcium channel blockers [12].

Another important feature of BECs is the limited transcytotic vesicles that restrict the diffusion and the transport of most hydrophilic molecules into the brain except certain molecules that utilize carrier-mediated transporters (CMT) 


\section{Article highlights}

- The blood brain barrier (BBB) is the single most important limiting factor that restricts the access of more than $98 \%$ of pharmaceutical drugs to the brain.

- Brain endothelial cells (BECs) are unique for being interconnected by tight junctions that seal spaces between BECs and have very limited transcytotic vesicles and therefore, restrict the transport of hydrophilic molecules to the brain.

- BBB functions and structure are not fixed, but in a dynamic change to respond to CNS needs during natural development and disease conditions. Changes to BECs structural features and transporter systems are widely reported in several brain disorders and contribute to BBB hyperpermeability.

- Lesioned brain is defined as an injury or disease in an area of the brain due to infection, problems with immune system, injury, disruption in blood supply, tumor, etc.

- Despite the reported disruption to BBB in different models of lesioned brain, a little or no increase in permeability to small therapeutic molecules (< 1000 Dalton) has been observed.

- Recent studies using nanoparticles-based delivery systems showed clearly that it is possible to selectively target the lesion area in the brain when injected intravenously in preclinical animal models. These observations suggested that the selective brain accumulation is mediated by BBB hyperpermeability, a phenomenon that could be very similar to the well-known enhanced permeability and retention (EPR) effect observed in solid tumors.

This box summarizes key points contained in the article.

(Figure 1) [8]. This explains how glucose, electrolytes, vitamins, and some peptides have the ability to cross the brain at 10-100 times faster rate than would be predicted based on their physicochemical characteristics [5]. A classical example of CMT is the glucose transporter (GLUT1) that facilitates bidirectional diffusion of molecules down a concentration gradient from a high-to-low concentration. An example of CNS drugs that enter the brain by this pathway is the anti-Parkinsonian drug L-dihydroxyphenylalanine ( $L$ DOPA) that utilizes large-amino acid transporters 1 (LAT-1) to get access into the brain [13].

Receptor-mediated transporters (RMT) facilitate the transport of specific molecules into and/or outside the brain (Figure 1). The source of insulin in the brain is coming from peripheral origin. Insulin gets access to the brain through insulin receptor (IR) that facilitates endocytosis from blood to the brain only [14]. Similarly, transferrin (Tf) transport to the brain is mediated by Tf receptor (TfR). TfR is expressed on both luminal and abluminal surface of BECs and therefore, it mediates both the endocytosis of TfR from blood to brain direction, in addition to the efflux transfer of apotransferrin from brain back to the blood $[15,16]$. In contrast, FC receptor allows unidirectional transport of lgG molecules from brain to blood [17].

Highly charged molecules or molecules that can bind to glycoproteins are likely to enter the brain through vesiclebased processes known as adsorptive endocytosis/transcytosis. These vesicles are either trafficked to lysosomes or routed all the way from luminal cell membrane to abluminal membrane giving a route to the passage of substances across the BBB. An important feature of this process is that it is different from classical saturated transporters in a way that increasing the concentration of ligands can further enhance the uptake. In fact, in extreme cases, this pathway can result in BBB damage due to coalescing of vesicles to form channels through the BECs [18]. Tat peptides act through this mechanism due to their highly charged nature [19].

Despite getting access into the brain, CNS drug delivery can still be compromised by the polarized expression of efflux transporters that shuttle out many drug molecules from the brain back into the circulation. These can be either energy dependent that belong to ATP-binding cassette $(A B C)$ gene family such as $P$-glycoprotein ( $\mathrm{P}-\mathrm{gp}$ ) and breast cancer resistance protein (BCRP) or energy independent that belong to the solute carrier transporter gene family such as organic anion transporters polypeptides (OATPs/Oatps). $\mathrm{P}$-gp, which is known as multidrug resistance, has received excessive attention, but it is just one of the many efflux transporter in the $A B C$ gene family. Efflux transport processes could be considered as natural protective mechanisms of the brain specially designed to prevent brain exposure to drugs and foreign molecules [20]. Thus, defect in efflux transporters can contribute to diseases, as in the case of reduced brain-to-blood efflux of amyloid $\beta(A \beta)$ peptide that contributes to elevated $A \beta$ levels in the brain $[21,22]$. P-glycoprotein has a very broad substrate profile and thus considered a formidable obstacle to CNS drug delivery. These include but not restricted to calcium channel blockers, antibiotics, cardiac glycosides, anticancer drugs, anti-epileptics, antidepressants, and immunosuppressants [23]. Example of marketed drug that act by inhibiting efflux transporters is verapamil for seizure treatment. Given the broad nature of these inhibitors, long-term use of efflux inhibitors can result in interference with endogenous substances and may cause neurotoxicity [23].

Furthermore, BECs are enclosed by and in continuous cross talk with a variety of cells (Figure 1). The abluminal surface of BECs is supported by pericytes and surrounded by $20-200-\mathrm{nm}$ thick vascular basement membrane (BM) composed of threedimensional networks of proteins predominantly collagen IV, laminins, nidogen, and heparan sulfate proteoglycans secreted by vascular and neural cells [24,25]. The composition of the $\mathrm{BM}$ is largely dependent on the location of the blood vessels. Large parenchymal arteries and arterioles are surrounded by two principally different BMs, the endothelial and parenchymal BMs. Patchy distribution of endothelial BM is observed surrounding postcapillary venules and this correlates with sites of lymphocytes extravasation [26]. This structure is further encased by astrocytic end feet cellular processes that ensheathe the blood vessels as well as neuronal synapses [27]. The association of endothelial cells, neural cells, and extracellular matrix (ECM) forms the neurovascular unit that plays a crucial role in modulating BBB integrity and drug penetration into the brain [5]. These structural features allow BECs to adapt constant cross talk with astrocytes, microglia, neurons, pericytes, and peripheral immune cells in order to refine $B B B$ function to serve the brain needs [5].

BBB structure presents both physical and biochemical barrier that significantly limit the entry of therapeutic molecules into the brain and, therefore, reduce treatment efficacy [28]. Figure 1 summarizes the structural features of BBB in healthy conditions and the mechanisms of molecular 


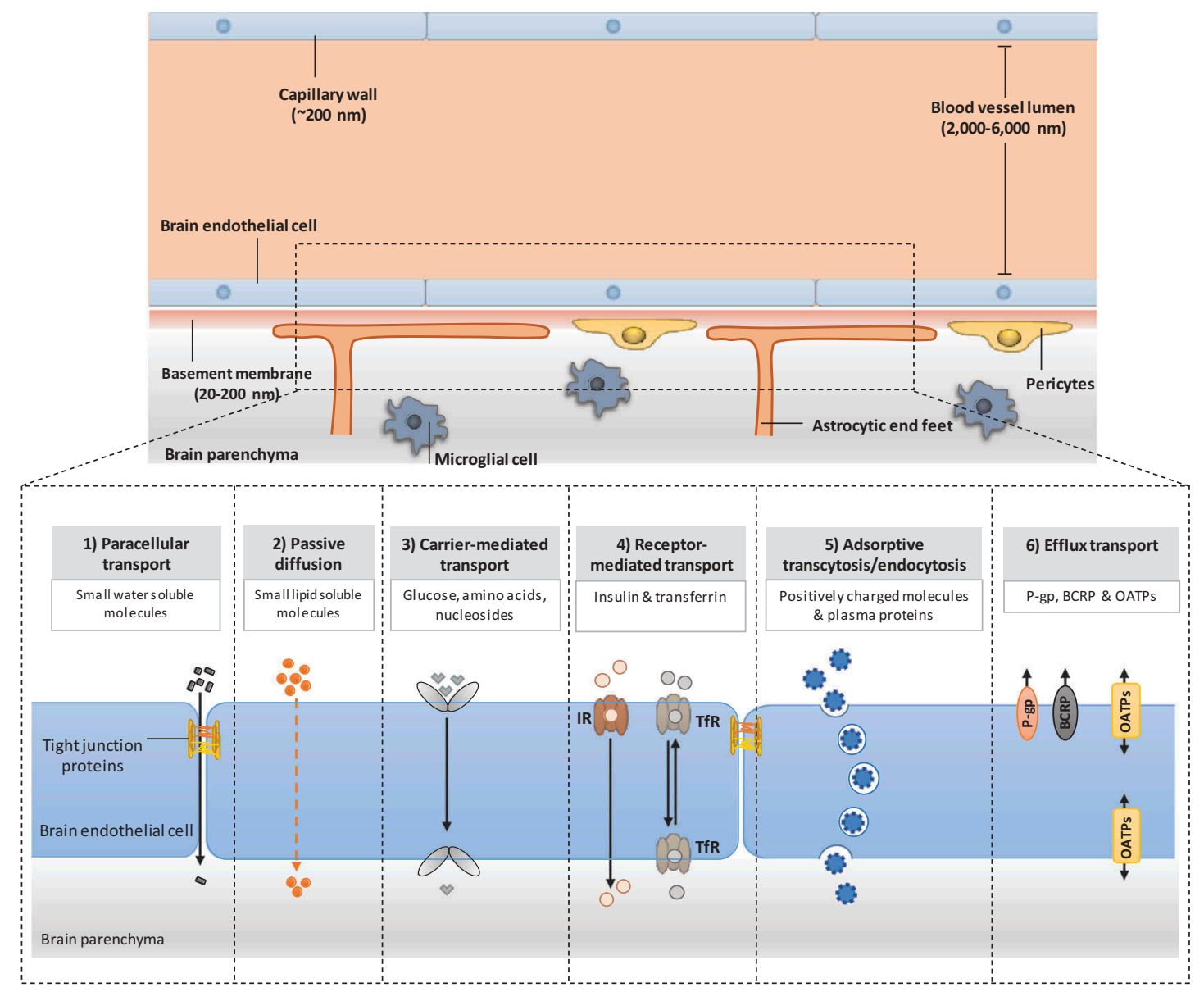

Figure 1. The BBB is situated at the walls of the blood vessels that supply blood to the central nervous system. The BBB is composed of a network of different cells that form the neurovascular units. These include astrocytes, microglia, pericytes, neurons, and brain endothelial cells with highly regulated tight junctions. Because of this complex structure, the transport of molecules through BBB is highly restricted to specific mechanisms that would apply to very limited molecules. 1) CNS delivery of large and hydrophilic molecules is almost entirely excluded by the physical presence of tight junctions; while 2) lipid-soluble molecules can diffuse across the brain endothelial cell passively. Other possible mechanisms to cross BBB include; 3); carrier-mediated transport (specific for certain molecules such as glucose, 4) receptor-mediated transport (e.g. insulin through insulin receptor (IR) and transferrin mediated by transferrin receptor (TfR), and 5) adsorptive transcytosis/ endocytosis for the transport of cationic molecules. 6) Despite getting access into the brain, CNS drug delivery can still be compromised by the polarized expression of efflux transporters that shuttle out many drug molecules from the brain back into the circulation (e.g. P-gp, OATPs).

transport across the $\mathrm{BBB}$. Apart from the restrictive role of $\mathrm{BBB}$, the compromised CNS drug delivery could also be related to drug factors. Among these are the drug molecular weight, the drug solubility, and the surface properties (such as the charge) [29]. Brain-blood ratio is used as an indication of the drug access into the brain. A ratio exceeding 1.0 indicates that the drug molecules have free access into the brain. On the other hand, a ratio between 0.3 and 0.5 represents sufficient brain access and a ratio of less than 0.1 indicates no access [20]. Based on the above, it is very clear that the BBB is the single most important factor limiting the treatment of lesioned brain. In fact, it has been estimated that BBB restricts brain access of more than $98 \%$ of therapeutic molecules.

\section{Strategies to enhance CNS drug delivery}

Several approaches have been principally investigated to enhance the prospective BBB penetration of therapeutic molecules. The rationale for these approaches is largely variable and can be summarized by the following, taking advantage of certain transport system, enhancing transmembrane diffusion, increasing enzymatic resistance, and improving pharmacokinetic profile. Care must be taken when considering structural modification as this can often results in unexpected observations. Although this area of drug development is very promising, it is at the same time very difficult and challenging as the structural modification processes can often interfere with the transport machinery of interest resulting in drug-brain penetration through different routes. These strategies have been thoroughly reviewed before $[5,12,13]$; however, below is a brief summary of the different categories implemented.

\subsection{Intracerebral administration}

For more specific and effective delivery of therapeutics to specific regions of the brain, direct injection/implantation into the region of interest using stereotactic coordinates and/or continuous intraventricular infusion of the drug into the brain tissue are usually applied. Promising results have been reported using 
this approach in clinical trials for Parkinson's disease for example [30,31]. Although this method is clearly the most effective in overcoming the BBB obstacle, there is a potential risk of several unwanted side effects such as damage to the healthy brain tissue along the needle track, CNS toxicity due to excessive drug concentration, susceptibility to infections, and general risks associated with anesthesia and surgery [32-34].

\subsection{Intrathecal injection}

Other less invasive approaches currently in clinical use include the direct injection into the cerebrospinal fluid (intrathecal injection). There are very few drugs that approved for such approach and the drug site of action here is the CNS surface rather than the brain parenchyma. Intrathecal chemotherapy is in clinical use for prophylaxis and treatment of CNS involvement by lymphoma or leukemia [35].

\subsection{Intranasal route}

This approach utilizes the nose submucous olfactory region as a potential route of drug transport from the nasal mucosa into the brain [36]. Several parameters need to be considered for this approach: (1) the capability of drug to transport across the nasal epithelium, (2) drug penetration through the arachnoid membrane that separates nasal submucous region from olfactory (cerebrospinal fluid) CSF space. Thus, the properties that control drug transport from nose to olfactory CSF are very close to those determining drug transport across the BBB. Based on that, small lipophilic molecules can best benefit from this approach in the absence of local disruption to the nasal mucosa $[36,37]$.

\subsection{BBB disruption}

Besides the direct intracranial administration of therapeutics to the brain, there have been considerable efforts to enhance CNS drug delivery by applying transient BBB disruption techniques. A very widely used technique in this area is the intracarotid arterial infusion of 2 molar concentration of mannitol that has poor diffusion into the brain. Mannitol is a hyperosmotic solution that causes the endothelial cells to shrink and, therefore, stretches the TJs. This approach commonly used to enhance delivery of chemotherapeutic drugs to brain tumors; however, the success of this technique can be dependent on the differential sensitivity of the blood-tumor barrier to disruption compared to the surrounding healthy brain tissues [38]. The use of ultrasound waves to open pores in the BBB is another area of active research [9]. The frequencies of ultrasound waves used for this purpose are $28 \mathrm{kHz}$ to $8 \mathrm{MHz}$ for rodents studies; however, the recommended frequencies for clinical use are $0.2-1.5 \mathrm{MHz}$ [32]. Thus, intravenous injection of microbubbles is typically used to reduce the power necessary to disrupt the BBB [39]. BBB disruption by this method is usually transient that lasts usually few hours to $<1$ day, depending on the brain region, provided that no permanent tissue damage occurs [40].

\subsection{Inhibition of $B B B$ efflux transporters}

Efflux transport systems (e.g. P-gp, BCRP) act at the luminal surface of EC to expel molecules back into the circulation and, therefore, represent a major restrictive feature of BBB against CNS drug delivery as explained earlier. Consequently, inhibition of those efflux mechanisms would therefore offer great opportunity to increase the amount of drug that reaches the brain. However, the beneficial effect of this approach is controversial in terms of safety and efficiency due to the broad nature of these inhibitory molecules. Therefore, recent efforts have been directed toward targeting discrete signaling pathways to have specific action rather than global inhibition of these efflux pathways. For more details, please refer to recent excellent reviews that cover this in more details [12,41].

\subsection{Molecular modifications}

Early efforts of structural modification of compounds were directed toward increasing lipid solubility to enhance passive diffusion across the BECs. However, studies over the years have proved that this approach is difficult to implement. Increasing the lipophilic nature of drug molecules, for example, can, on the contrary, restrict the ability to reach the CNS because of increased uptake by peripheral organs or entrapment within the lipid membrane [42]. In fact, until now, there is no FDA-approved CNS drugs that have increased CNS accumulation by enhancing lipid solubility. Thus, efforts have been shifted toward the use of medicinal chemistry to enhance brain uptake of water-soluble drugs through the adoption of BBB influx transporters to enhance CNS drug delivery. Currently, there are two main trends in this adoption process: first, targeting RMT such as BBB IR or TfR through engineering of bispecific antibodies or molecular Trojan horses. Second, engineering drugs have affinity to an endogenous BBB CMT system that normally facilitates the entry of peptides, vitamins, or hormones into the brain. To enhance drug transfer by RMT, conjugation to peptidomimetic monoclonal antibody (mAb) that can traverse the BBB through specific receptor is preferable to the use of endogenous substrates such as insulin or TfR. This is to avoid any unwanted slide effects (hypoglycemia in the case of insulin) or competition on the binding site (high concentration of endogenous TfR $25 \mu \mathrm{mol} / \mathrm{L}$ ) [37]. EPO is a neurotrophic factor that has limited brain penetration. Reengineering EPO by conjugation with human IR (HIR) mAb produced a brain-accessible form of EPO at $2.1 \%$ of ID/100 g of brain [43]. The enhanced brain delivery through CMT was discovered serendipitously for anti-Parkinson drug L-DOPA that does not cross the brain by passive lipid diffusion. LDOPA on the other hand is a large neutral amino acid that utilizes LAT1 to cross the BEC. Once in the brain, L-DOPA is decarboxylated to the active form dopamine $[44,45]$. Gabapentin is another example of water-soluble drug that gets access into the brain through LAT1 system [46]. OATPs also play an important role in the transport of opioid analgesics and pain management [47]. Care must be taken to consider alterations in the pharmacokinetic profile of antibodies after the reengineering process, the possibility of clearance by peripheral organs expressing these receptors and changes in 
the influx transporters expression in different neurological disorders.

\section{Modifications of the BBB in lesioned brain}

It is now well established that BBB functions are in dynamic response to CNS needs during natural development and disease conditions. These changes involve both the structure and the transporter systems, with the latter being more sensitive in many pathological conditions. BBB disruption is a serious complication in many diseases of the CNS as summarized in Table 1. This could be a result of extrinsic factors (multiple sclerosis [MS] and [48,49] meningitis [50]) or due to intrinsic factors as in the case of ischemic stroke [51-53], Alzheimer disease (AD) [54,55], traumatic brain injury (TBI) [56], seizures [57,58], diabetes [59], chronic pain [60], and many others [61]. In many of these disorders, the damage to BBB is clearly a secondary effect to the primary insult as in the case of TBI and stroke; however, in other conditions as in the case of MS and $A D$, it remains unclear if $B B B$ disruption has a role in the disease initiation process [24]. Most of the research has focused on the role of the TJ as the main contributor to BBB disruption. However, enhanced expression of other transcellular pathways can happen as well and may even be the dominating factor to BBB disruption $[62,63]$.

During ischemic stroke, there is a strong evidence from preclinical $[62,63,66]$ and clinical $[67,68]$ studies that BBB integrity is impaired. Compromised BBB function leads to a biphasic increase in permeability and uncontrolled entry of molecules into the brain. In normal conditions, endothelial cells, through tightly regulated transcellular transport functions and TJ proteins [63], are the primary regulators of the entry of blood-borne molecules into the brain (Figure 2). The contribution of these two pathways to BBB hyperpermeability after ischemic stroke is controversial [56]. However, the most accepted model of hyperpermeability is early phase (within
$6 \mathrm{~h}$ ) increase in endothelial vesicles (termed caveolae), followed by TJ proteins disassembly and loss of integrity at a later point ( $>24 \mathrm{~h}$ poststroke). This model is supported by recent evidence from transgenic mouse strain study in which endothelial TJs were labeled with eGFP, allowing the dynamics of TJs to be monitored in vivo in real time [63]. This model is also supported by recent evidence from rats ischemic stroke model, which proved that BBB opening to macromolecules (transcellular route) precedes permeability to small ions (paracellular opening) [69]. Moreover, the reported BBB disruption is significantly exacerbated when combined with other comorbidities; however, the consequence of events contributing to BBB hyperpermeability has not significantly changed [62].

Similarly, MS lesions are characterized by severe inflammation, loss of barrier, and infiltration of monocytes [70]. Disruption of the BBB has been proved in preclinical in vivo studies of both acute and chronic MS using gadolinium (Gd) contrast enhanced MR $[71,72]$. In MS, BECs are phenotypically modulated to express endothelial cell adhesion molecules that enhance the inflammatory cells migration through the disrupted BBB. Infiltration of the brain by inflammatory cells triggers MS inflammatory cascade through the production of inflammatory cytokines and chemokines (e.g. IL $1 \beta$, INFY, and TNFa) within the cerebral interstitium. In addition, BBB disruption in MS has also been reported to involve both structural remodeling and reduced expression of TJ proteins [73].

In addition to the mechanisms described above, the loss of $\mathrm{BBB}$ integrity can also be a result of upregulation of proteolytic enzymes that cause the degradation of vascular BM and ECM proteins. The precise mechanisms behind that are not fully elucidated; however, the significant upregulation of matrix metalloproteinases (MMPs) and plasminogen activator systems are the main triggers. Different enzymes or cytokines have been reported before to activate MMPs through cleavage of pro-domains into the active forms including membranetype 1-MMP (MT1-MMP) and plasmin. Moreover, the high

Table 1. Pathological conditions with BBB disruption.

\begin{tabular}{|c|c|c|}
\hline Disease & Evidence of BBB disruption from clinical and preclinical studies & References \\
\hline \multicolumn{3}{|l|}{ Extrinsic factors } \\
\hline MS & $\begin{array}{l}\text { - Gd-enhanced MRI } \\
\text { - Immune cells infiltration } \\
\text { - Loss of TJ protein in postmortem samples }\end{array}$ & {$[48,49]$} \\
\hline Infections - e.g. meningitis & - Enhance proteases activity (e.g. MMP9) and immune cells infiltration & [50] \\
\hline $\begin{array}{l}\text { Intrinsic factors } \\
\text { Ischemic stroke - hypoxia, glial activation, and } \\
\text { macrophages infiltration }\end{array}$ & $\begin{array}{l}\text { - Neuroimaging } \\
\text { - Immune cells infiltration } \\
\text { - Upregulation of E-selectin and P-selectin }\end{array}$ & [51-53] \\
\hline $\mathrm{TBI}$ - edema and hemorrhage & - Biphasic increase in BBB permeability & [56] \\
\hline Small vessels disease - e.g. diabetes & - Pericytes loss due to oxidative stress mediated by high glucose level & [59] \\
\hline$A D$ & $\begin{array}{l}\text { - Increase in BBB permeability measured by CSF albumin levels was observed in AD patients } \\
\text { and correlated with disease progression } \\
\text { - Decrease the levels of amyloid } \beta \text { efflux transporter from brain to blood such as Pgp and LRP1 } \\
\text { in patients with AD } \\
\text { - Inflammation-induced pericytes loss and disruption to BBB }\end{array}$ & {$[5,54,55]$} \\
\hline
\end{tabular}

LRP1: Low-density lipoprotein receptor-related protein 1; BBB: blood-brain barrier; AD: Alzheimer disease. 


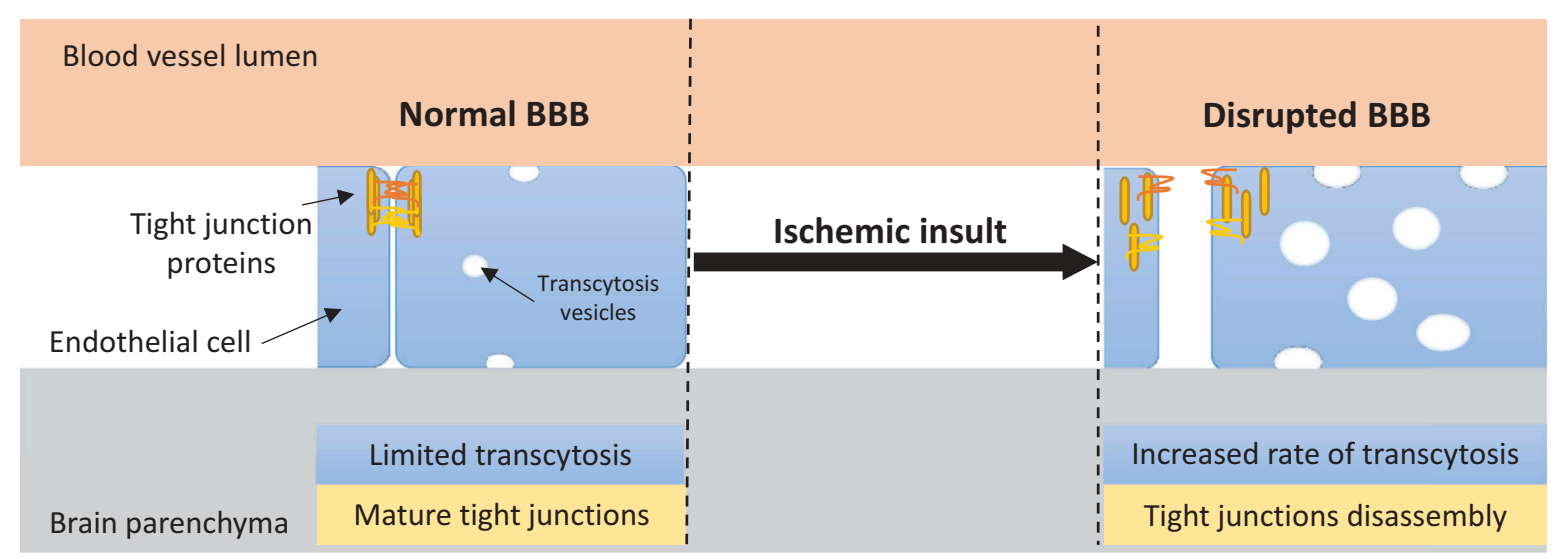

Figure 2. Structural changes to BBB after ischemic stroke: Several experimental ischemic stroke models reported qualitative and quantitative increase in transcytotic transport across the BBB and tight junction strands disassembly. These structural changes contribute to the BBB hyperpermeability reported after ischemic stroke.

level of inflammatory cytokines and reactive oxygen species produced after certain neurological conditions (e.g. stroke) can stimulate MMP9 section and activate pro-MMP9 [50,74].

Because of BBB disruption, leakage of plasma products and infiltration of immune cells (monocytes, neutrophils, and macrophages) into the brain parenchyma will occur which can further aggravate the BBB damage by secreting proteases and inflammatory cytokines. Consequently, ECM receptors expression is altered, and this has a crucial effect on the homeostasis of NVU cells such as survival, proliferation, and migration [74]. These changes explain the loss of the cells in contact with the vessels due to the loss in receptors that anchor these cells in place. In experimental model of ischemic stroke, microvasculature coverage of astrocytes gradually reduced to $74 \%$ in $4 \mathrm{~h}, 16 \%$ in $12 \mathrm{~h}$, and almost none $48 \mathrm{~h}$ after ischemia [75]. This also associated with migration of pericytes away from blood vessels breaking through BM that surrounds them [76]. On the contrary, microglial cells were observed to migrate in close proximity to the microvasculature [77].

It can, thus, be inferred from above that there are several mechanisms by which the BBB can be compromised in lesioned brain, thus highlighting the possibility that diseased BBB can itself act as a gate for therapeutic access. For this review, I would like to discuss the role of BBB hyperpermeability to enhance selective drug accumulation in the brain by considering two pathological conditions, stroke and MS, since several promising examples have been demonstrated with these two conditions before. An effective drug delivery approach in those situations should be able to selectively penetrate areas of BBB hyperpermeability as compared to other brain regions where BBB permeability is unaffected.

\section{How to take advantage of BBB disruption to enhance drug delivery to the brain?}

Pharmacological transient disruption of the BBB is a widely used strategy to enhance drug delivery to the brain. A traditional example is through the osmotic opening of BBB using mannitol that is used in the treatment of certain CNS cancers [78] and the use of ultrasound waves to open transient pore in the BBB to enhance drug penetration [79]. The downside of such strategies lies in the lack of selectivity meaning that not only the potential therapeutics could get access into the brain but also any other circulating substances that are normally excluded by the BBB can get access to the CNS with potentially harmful consequences.

Recent studies have shown that pathological BBB disruption in models of Alzheimer's and MS resulted in little or no increase in permeability to small therapeutic molecules $(<1000 \mathrm{Da})$ [80]. This observation could also be true for therapeutic approaches of BBB disruption, since, in these situations, the disrupted BBB could still retain some degree of barrier selectivity. This, in addition to the suboptimal pharmacokinetic nature of many drug molecules, means that attempting to therapeutically disrupt the BBB can have uncertain consequences.

Based on the above, the dilemma of therapeutic drug delivery in lesioned brain can be stated in two parts: (1) currently, it is proven that BBB is disrupted in lesioned brain and this has serious consequences on pathological evolution, and (2) the BBB disruption does not improve the delivery of low molecular weight molecules into the diseased brain tissue.

Since conventional drug delivery methods have very limited access to the brain, systemic high doses are often required for a drug to reach therapeutic levels in the lesioned CNS. This carries the obvious risk of collateral damage due to unnecessary exposure of normal healthy tissues.

\section{Promising drug delivery approaches in other conditions: the cancer story}

Over the past few decades, nanotechnology-based drug delivery systems proved to offer superior therapy control compared to small molecule drugs in several pathological conditions [81,82]. Cancer therapy [83-85] and inflammatory diseases $[86,87]$ are the most promising applications so far, where several formulations have already made it into clinical practice [88]. Among those drug delivery systems, liposomes and other polymeric nanoparticles have a great potential since they dramatically change the pharmacokinetics and the pharmacodynamics profiles of the encapsulated drugs, reduce the drug-associated toxicity, and, therefore, improve the patients' experience. An important parameter behind the improved 
therapeutic outcome of these delivery systems is the increase in blood circulation time. AmBisome ${ }^{\circledR}$ and Doxil ${ }^{\circledR}$ are examples. In the case of AmBisome (liposomal amphotericin), the long blood circulation is due to the rigid lipid nature of the liposomal formulation [89]. On the other hand, the long circulation time of Doxil ${ }^{\circledR}$ (liposomal doxorubicin) is provided by surface coating of nanoparticles with the hydrophilic polymer polyethylene glycol [90]. As a result of the long blood circulation, there is a greater chance to accumulate passively in tumor tissues as a result of the latter's hyperpermeable blood vessels and impaired lymphatic drainage. This phenomenon is collectively known as the enhanced permeability and retention (EPR) effect $[91,92]$. This effect was first described by Maeda et al. [91] and since then, it is believed to be behind the selective targeting of the tumor and inflammatory tissues. The observed hyperpermeability has been shown to be mediated through one or more of the following pathways: endothelial membrane fenestrations [93], gaps in the blood vessels [93], and the transcellular vesicular transport system (caveolae) [94].

Similarly, hyperpermeability of BBB is widely reported in preclinical and clinical studies in several models of lesioned brain [5]. Recent studies in middle cerebral artery occlusion model of ischemic stroke reported BBB hyperpermeability $[62,63,69,95]$ due to a qualitative and a quantitative increase in transcytotic transport across the BBB in the early phase after ischemia in addition to more than $30 \%$ opening of TJ strands that usually follow at later stages [63]. Based on the above, there is a clear resemblance between the BBB endothelial cells structural adaptation in lesioned brain and what has been reported in cancer and inflammatory conditions. This shows that nanotechnology drug delivery systems can be equally efficient in achieving selective and enhanced drug delivery to the lesioned brain as compared to what is seen in cancer and inflammation. It is important to mention here that for brain tumors, the beneficial role of EPR effect to enhance drug delivery is controversial. This is because EPR effect is usually observed at late stage of brain tumor development and it is significantly weaker in the cranial microenvironment compared to peripheral cancer. Thus, EPR effect may enhance drug delivery to the brain; however, due to the late stage of brain tumor, passive targeting alone is insufficient for effective treatment $[96,97]$.

\section{Promising examples on selective drug delivery systems to the lesioned brain}

Recent studies using nanoparticles-based delivery systems for the treatment of brain conditions showed clearly that it is possible to selectively target the lesion area of the brain when injected intravenously in preclinical animal models [98-101]. These observations suggested that the selective brain accumulation could be mediated through the increase in the permeability of the BBB endothelium, a phenomenon that could be very similar to the well-known EPR effect observed in cancer.

These findings have been supported by imaging data that measure the total accumulation of drug delivery systems into the brain. Of these, I would like to highlight the detection of
[18F]-labeled liposomes with sizes between 100 and $200 \mathrm{~nm}$ into a rat model of ischemic stroke using PET imaging [102] and fluorescently labeled liposomes using ex-vivo optical imaging [100]. These liposomes did not show any accumulation into the healthy brain tissue, due to the presence of an intact BBB in the normal areas. In one study, liposomal FK506 of $100 \mathrm{~nm}$ diameters was injected systematically at the reperfusion time $1 \mathrm{~h}$ after the start of ischemia in a rat model of stroke (Figure 3). The accumulation of liposomes in the lesioned brain was visualized ex-vivo by detecting the fluorescence signal of the liposomes using optical imaging. The areas of liposomal accumulation co-localized with infarcted area as detected by 2,3,5-triphenyltetrazolium chloride stain. As FK506 (tacrolimus) is a neuroprotectant drug, rats treated with this technology were shown to have lower levels of apoptosis and inflammation, correlating with an improved motor function [100].

Consistent with the assumption that selective accumulation of those drug delivery systems into the lesioned brain is mediated by BBB hyperpermeability, the size of those delivery systems has been reported to have great influence on crossing the damaged BBB. Cruz et al. demonstrated that polylactic-co-glycolic acid nanoparticles of different sizes showed different permeabilities into the brain following TBI model in mice. The sizes tested were approximately 100, 200 , and $800 \mathrm{~nm}$, injected systemically at the time of brain injury. As expected, the 100-nm particles showed the highest penetration into the injured area of the brain. The penetration was less with the 200-nm particles and least with the 800-nm particles [103]. Similarly, Fukuta et al. followed the accumulation of lipid nanoparticles injected intravenously shortly after induction of ischemic stroke in rats. Nanoparticles of approximately $100 \mathrm{~nm}$ accumulated to a higher extent compared to those of $200 \mathrm{~nm}$, while lipid nanoparticles of $800 \mathrm{~nm}$ did not accumulate in the ischemic brain regions [104].

The results of the selective increase in the accumulation of drug molecules into the lesioned brain area after encapsulation into nanoparticle-based delivery systems were translated further to improve the therapeutic effect and functional outcome. In the case of ischemic stroke, for example, these approaches could be classified into the following categories: (1) supplement therapies such as the delivery of ATP and oxygen that are crucial for neuronal cell function and survival [105-110]; (2) neuroprotective agents including most of the anti-inflammatory, immunomodulatory drugs, and free radical scavenging agents aiming to reduce brain damage and edema formation [100,104,111-114]; and (3) thrombolytic agents that aim to restore blood supply to the ischemic areas of the brain [115-117]. Please note that for thrombolytic agents, the drug will work on dissolving the clot in the brain vasculature and therefore, crossing the BBB is not required in this case. Among these categories, the use of neuroprotective agents has been widely tested. In a general description, those agents are molecules that aim to increase the cell resilience or recovery under disease conditions and therefore minimize brain damage expansion. Although these molecules are quite promising in concept, they failed clinical translation in more than 100 phase III clinical trials with no clear added benefits in therapeutic outcomes [118-120]. There are several reasons 

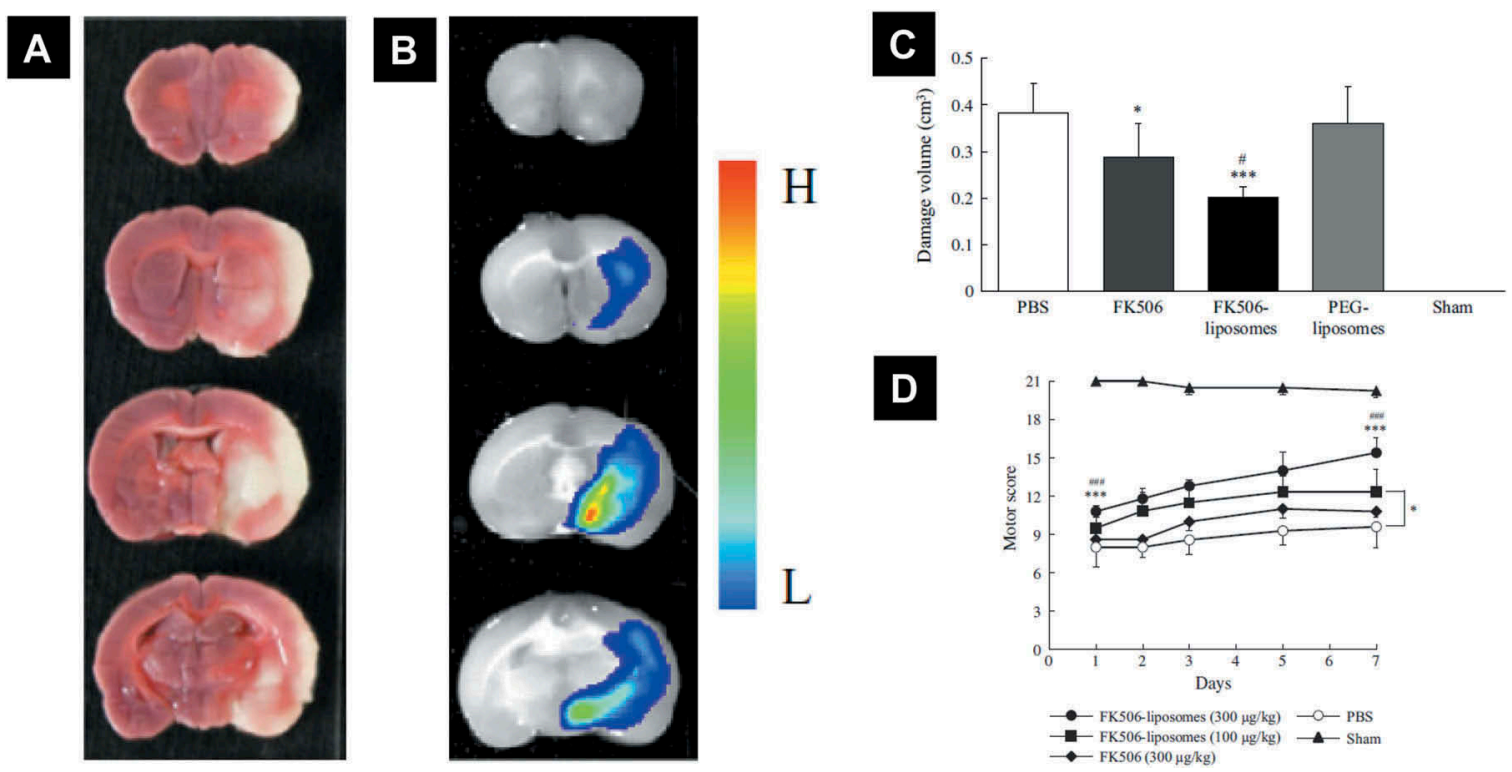

Figure 3. Liposomal accumulation into rat model of ischemic stroke. PEGylated liposomes encapsulating the neuroprotective drug FK506 (FK506-liposomes) were intravenously administered in a rat model of ischemic stroke. Stroke was induced by middle cerebral artery occlusion (MCAO) for $1 \mathrm{~h}$. A) The damaged area in the brain can be seen as paler regions on brain slices (right side) stained with TTC dye. B) In this study, FK506-liposomes were injected before the recovery of cerebral blood flow to the brain and their selective localization in the ischemic area (right side) was followed ex-vivo by optical imaging of fluorescently labelled liposomes. C) Liposomal delivery of the neuroprotective drug FK506 significantly suppressed the expansion of brain cells damage and D) significantly improved the motor function score. Reproduced with permission from reference [100].

behind those disappointing results; however, the poor BBB translocation is, by no doubt, a crucial limiting factor.

Encapsulating therapeutic molecules into delivery systems such as liposomes consistently displayed a better effect on infarct volume reduction and motor recovery in animal stroke models, and repeatedly showing selective accumulation in the ischemic regions of the brain. An example on that is liposomal fasudil, given directly after ischemic reperfusion to suppress neutrophil invasion into the lesioned brain. The therapeutic effect of this formulation was significantly higher compared to free drug which reemphasize the importance of the drug delivery system to provide access to the lesioned brain and maintain appropriate drug concentration in that area [104]. The natural free radical scavenger enzyme superoxide dismutase (SOD) is another neuroprotective agent that showed better therapeutic activity for stroke after encapsulation into liposomes. SOD helps to reduce permeability changes and lipid peroxidation following free radical production after cerebral ischemia. However, in its free form, SOD has very limited brain penetration due to its inability to cross the BBB and its very short half-life $(<6 \mathrm{~min})$. Therefore, encapsulation into nanosized drug delivery platforms was required to enhance the antioxidant activity in the brain. In vivo experiments following systemic administration of SOD-loaded liposomes demonstrated a significant increase in the enzymatic activity in the brain and provided a significant protection against free radicals that reflected in infarct size reduction. The high SOD activity observed in the infarct region suggested being due to the increase in liposomal SOD transport mediated by BBB disruption. It is hypothesized that SOD-liposomes neuroprotective effect is mainly exerted on the endothelial cells; however, this might be extended to include neurons and microglia as well since liposomal extravasation to brain parenchyma is excepted [121-124]. Liposomal drug delivery has proved its effectiveness in improving brain delivery and therapeutic efficacy of citicoline (CDP-choline, cytidine-5-diphosphocholine) $[98,114,125-129]$. Citicoline is an essential intermediate in the synthesis of phosphatidylcholine (a major component of neuronal membranes). Preclinical studies demonstrated that pharmacological neuroprotective effect of citicoline is mediated through the stimulation of the brain phospholipids synthesis, inhibition of phospholipase A2 (PLA2) activation, and oxidative stress reduction [130]. However, the clinical beneficial effect of citicoline is highly debatable as several large populations trials reported unclear outcomes $[98,131]$. This is due to the polar nature of citicoline, which restrict its transport through BBB [114]. A noticeable reduction in infarct volume $(\sim 30 \%)$ was observed from liposomal citicoline as compared to free citicoline [114]. Similar to previous studies, the improved therapeutic effect observed from citicoline liposomes is hypothesized to be related to selective accumulation into the brain as a result of BBB disruption [129]. The beneficial therapeutic effect of CDP-choline was also confirmed recently with serial MRI imaging in rat model of MCAO over 7 days [114].

Promising therapeutic benefits have also been reported in other models of lesioned brain such as in MS using long circulating liposomal drug formulation encapsulating methylprednisolone. In this study, Turjeman et al. compared the therapeutic activity of liposomal prednisolone to that of the free drug after multiple injections into a mouse model of MS. Treatment was started at the onset of clinical signs (day 8 after T-cell transfer) and continued for 3 days. Although both treated groups showed better disease improvement compared to the control group, therapeutic benefits from liposomal formulation exceed those of the free drug. That was translated into a significant reduction in disease score, duration, and mean 
burden. The therapeutic benefits observed from the liposomal formulation of prednisolone compared to the free drug are most likely due to the selective passive accumulation of liposomes into the lesion site taking advantage of BBB disruption. That was proved using Gd-based MR imaging [99]. However, it is important to remember here that BBB disruption in MS is highly variable and occurs most commonly during disease relapse $[73,132,133]$. Therefore, care must be taken in considering the beneficial effect of nanoparticle-based drug delivery in this case due to this variable window of opportunity.

The studies described above showed that passive targeting of delivery systems through damaged BBB is good enough to demonstrate a therapeutic effect; however, recent studies have also shown that further improvement could be gained if combined with active targeting. One example is the use of liposomes loaded with the neuroprotective drug asialo-EPO (AEPO), a potent antiapoptotic agent, with the aim to slow down the rate of programmed cell death across the infarct area. The liposomes were injected immediately after reperfusion and were shown to accumulate in the ischemic regions of the brain. These liposomes have the capacity to target neuronal cells through the EPO receptors. A single dose of AEPOliposomes was enough to show a significant decrease in the infarct volume [101] and to suppress the motor function deficit compared to untreated controls [134]. At day 7 after reperfusion, a significant recovery from motor function deficit and right forepaw paralysis was observed in the AEPO-liposomes-treated group [134]. Similarly, the therapeutic effect of CDP-choline liposomes could be further improved by formulating actively targeted liposomes against mannose receptor [125] or directed specifically to the peri-infarct region using anti-HSP72 liposomal citicoline [135]. Another example is the fluorescently labeled transferrin-targeted liposomal formulation that showed maximum accumulation into the brain when injected 1 day after cerebral ischemia. This is the time point where the highest transferrin receptor expression was observed. The accumulation of transferrin-targeted liposomes is most likely to be conveyed through receptor-mediated transcytosis process. However, the observation of liposomal extravasation into the brain parenchyma also suggests that it could be linked to BBB disruption at that time point [136].

The examples discussed in this section argue that using a drug delivery technology has a clear add-on benefit compared to the free drug. The exact mechanisms by which the selective brain localization happens are largely unknown and require in depth studies. BBB hyperpermeability (EPR-like effect), as an explanation, seems satisfactory particularly for long circulating-delivery systems; however, the improved efficiency observed from actively targeted systems compared to standard long circulating ones indicated that other factors can be involved.

An important parameter that has not been deeply investigated is the ability of those delivery systems to travel inside the brain parenchyma after crossing the discontinued BBB. The heterogenous nature of the BBB disruption and the presence of BM could act as a second barrier that limits the nanoparticles access from the blood to the brain. This phenomenon has been reported before by Moldoon et al. by studying the brain localization of two different types of iron- oxide nanoparticles: Feridex and MION. Both nanoparticles have comparable size but differ only in surface coating as Feredix is known to be opsonized quite quickly compared to MION. The distribution of those nanoparticles in the brain was assessed by MRI, after systemic administration of Feredix and MION following osmotic disruption of the BBB. It was very interesting to observe that at the histological level, both nanoparticles demonstrated very distinct distribution in the brain despite both showing homogenous brain distribution with MRI. Histological observation showed that Feredix nanoparticles were mainly trapped around the brain blood vessels in the space between BECs and BM; on the contrary, MION nanoparticles were found within cell bodies throughout the disrupted hemisphere [137]. This study emphasizes on the importance to complement bulk imaging techniques (MRI and PET) with histological analysis to assess cellular localization of the delivery system after crossing the disrupted BBB. Similar evidences were observed from three recent in vivo studies examined the capacity of nanoparticles to extravasate the brain parenchyma after induced entry into the brain by transient opening of BBB using focused ultrasound. These studies elucidated that the capacity of nanoparticles to travel inside the brain parenchyma is highly dependent on the size and surface properties of the nanoparticles that had a significant impact on their localization relative to the vascular BM [138-140]. Nanoparticles with small size and/or hydrophilic surface $(<120 \mathrm{~nm})$ have higher ability to travel away from the blood vessels compared to large and/or negatively charged nanoparticles. The barrier effect of BM described above is based on experimental evidence from studies performed in healthy animals where no changes in BM and ECM composition are expected. Little is known how BM and ECM modification in different brain lesions could impact nanoparticles distribution in the brain. This is a crucial point that deserves further investigation.

The release profile of drug delivery systems applied for treatment of brain disorder has not been systematically investigated either. Moreover, the actual release profile of drug molecules once in the brain has been hardly studied before. However, based on the knowledge generated from in vitro studies and other diseases like peripheral cancer, it is expected that drug release will be dependent on several parameters. These include the type of drug delivery system, diffusion rate of drug through the lipid bilayer (liposomes) or polymeric matrix (polymeric nanoparticles), biodegradable properties of polymers, the lipid composition and rigidity for liposomes nanoparticles, nanoparticles size, and the interaction with plasma protein (before getting access into the brain) and ECM proteins in the brain.

The toxicity related to the nanoparticle-based drug delivery systems in the context of brain delivery is still an area that requires more specific investigations. Most of drug delivery systems used for brain delivery have been tested before for other applications and thus, limited neurological toxicity is expected particularly for those already in clinical use such as liposomes and polymeric nanoparticles. The toxicity profile will be very much dependent on the nanoparticles composition, surface properties, and concentration. Previous studies using liposomes and polymeric nanoparticles have not 
reported severe toxicological changes following accumulation in the brain. Few studies reported the susceptibility to neurotoxicity and seizure following MR imaging with gadolinium in preclinical models. This effect was dose dependent and correlated with repeated administration [141].

\section{Benefits of nanoparticle-based delivery systems compared to other CNS drug delivery strategies}

Several approaches to enhance drug transport to the brain have been under development for the past few decades. Although enhanced BBB entry is the main aim for those approaches, other parameters are likely to be equally important. These include improved pharmacokinetic profile of the drug, achieving site-specific delivery, enzymatic stability, and reduced systemic toxicity.

Ligand-targeted drug conjugates have proved promising results to enhance brain delivery. Those are designed in away that the binding of the ligand will be on a region that is not directly involved in the binding of endogenous substrates. A promising example on that is the utilization of OATP/Oatp influx transport systems to enhance drug delivery for ischemic stroke. Statins (e.g. pitavastatin and rosuvastatin) are substrate for OATPs (Oatp1a4) influx system that is expressed on the brain microvascular endothelium [142]. OATP/Oatp isoforms have also a very important role in the brain delivery of opioid analgesic peptides (e.g. deltorphin II) for pain management purposes [143]. Interestingly, Thompson et al. have reported, in rat model of cerebral ischemia, that functional expression of Oatp1a4 is enhanced after hypoxia [144]. Moreover, microvascular expression and activity of Oatp1a4 at the BBB can be further increased through inhibition of TGF- $\beta$ signaling $[144,145]$. Since TGF- $\beta 1$ expression is known to be enhanced after cerebral ischemia [146], pharmacological inhibition of TGF- $\beta$ pathway could have very critical effect to enhance the function of Oatps influx transporter and ultimately increase CNS drug delivery. Despite showing promising results, care must be taken in the design and evaluation of therapeutics that act through these pathways (Table 2). First, ligand-drug conjugates should have desirable pharmacokinetic properties and maintain drug activity and ligand targeting capability. Second, ligand-drug substrates for one transport system e.g. OATP/Oatp can also transported by at least one additional transporter (e.g. P-gp efflux transporter) and thus, the overall accumulation in the brain may be reduced. Third, the broad nature of many of these transporters and the interference with endogenous substrates can also pose another obstacle toward clinical translation. Likewise, the exact mechanism of BBB permeation of drug-conjugates can be sometimes difficult to predict based on theoretical principles only. For example, attachment of glucose moiety to a peptide can improve the transport across the BBB, but this is not necessarily due to the peptide ability to use the BBB glucose transporter (GLUT1). Finally, ligand-drug conjugates can accumulate in different region of the brain (disease not specific) that may cause undesirable side effect. Moreover, changes in the expression of certain transport systems in the brain in some diseases have critical impact on the translocation ability of ligand-drug conjugates into the brain and perhaps cause heterogenous distribution $[42,147]$.

Nanoparticle-based drug delivery systems, on the other hand, have less impact on the pharmacological properties of the drug and the pharmacokinetic profile mimics that of the drug delivery system. The accumulation in the lesioned brain is principally selective to the regions of damaged BBB within the lesioned brain $[100,103]$. Therefore, this approach could have higher degree of disease specificity compared to ligandtargeted therapies. Additionally, nanoparticle-based drug delivery systems offer other benefits in terms of the drug stability and ability to modulate drug release [80]. However, in order for the drug to act on brain tissues, complete shuttling into the brain parenchyma needs to follow. Several parameters can affect this process such as (a) the BECs signaling pathways to allow complete transcytosis into the brain, (b) the ability of drug delivery systems to pass through the BBB BM and travel through the brain ECM. This hindrance effect has been demonstrated before with iron oxide nanoparticles [137]. However, due to the complex nature of ECM and the significant modification reported in many neurological conditions, in-depth studies are warranted to understand the exact nature of these limiting factors.

\section{Expert opinion}

Lesioned brain disorders such as cerebral ischemia, TBIs, and postoperative brain tumors represent a leading cause of death and disability worldwide. Yet, treatment options are extremely limited and represent an area of unmet clinical need. A significant problem with these disorders is the long-term disability that exceeds that caused by any other chronic illnesses. The resulting long-term disability means that those who survive may require long-term careers. As a result, the economic costs of those disorders are very high. For example, only the burden of stroke on economy is estimated to cost about $£ 8.9$ billion a

Table 2. Advantages and disadvantages of nanoparticles-based CNS drug delivery compared to ligand-drug conjugates

\begin{tabular}{|c|c|c|}
\hline Parameters & Ligand-drug conjugates & Nanoparticles-based CNS drug delivery \\
\hline Pharmacological activity & $\begin{array}{l}\text { Structural modification may affect pharmacological } \\
\text { activity of the final product }\end{array}$ & $\begin{array}{l}\text { No modification to the pharmacological properties is expected; } \\
\text { however, drug release is required }\end{array}$ \\
\hline Pharmacokinetic profile & $\begin{array}{l}\text { Pharmacokinetic profile of the final product can be } \\
\text { different to that of drug and targeting ligand }\end{array}$ & Mimics drug delivery system pharmacokinetic profile \\
\hline Undesirable effects & $\begin{array}{l}\text { Brain targeting is not specific to the lesioned-brain and } \\
\text { can be heterogenous/(disease nonspecific) }\end{array}$ & $\begin{array}{l}\text { Accumulation is mainly restricted to BBB damage area with in the } \\
\text { lesioned brain/(disease specific) }\end{array}$ \\
\hline Translocation into the brain & $\begin{array}{l}\text { Binding affinity must be retained to allow binding to BBB } \\
\text { transposers }\end{array}$ & $\begin{array}{l}\text { Shuttling through the BEC into the brain parenchyma is necessary } \\
\text { followed by the ability to travel through BM and ECM }\end{array}$ \\
\hline
\end{tabular}

BBB: blood-brain barrier; BEC: brain endothelial cells; BM: basement membrane; ECM: extracellular matrix 
year in the United Kingdom and with an ageing population, this figure is likely to increase significantly [148]. Therefore, there is an urgent need for efficient use of resources toward the development of cost-effective treatment of such conditions. The BBB is formed by specialized capillary endothelium that excludes more than $98 \%$ of small molecule neurotherapeutics and almost all large molecular drugs. Because of the poor accessibility of therapeutic molecules to the brain, there currently have very few effective treatments for most of the lesioned brain disorders [42]. The development of effective pharmacological treatment for lesioned brain is facing repeated translational failure, despite the tremendous success that has been made in molecular neuroscience [118]. As a matter of fact, many new neurotherapeutics that have been discovered carry the potential to treat many brain disorders, if an effective technology to cross the BBB is in place. Therefore, the development of new methodologies in this area can give new hope to those therapeutics.

The BBB plays an essential role in the stability and homoeostasis of the brain tissues. However, the BBB structure is in a dynamic change in several brain disorders. This leads to a high number of pathophysiological processes which contribute to the spread of damage over a longer period. Understanding the molecular basis and the time frame of such changes can bring upon new opportunities for brain drug delivery and open a new market for CNS therapeutics. This would not be accomplished without collaborative efforts between biological neuroscientist, drug delivery scientists, and clinicians to link those pathophysiological structural changes of brain endothelium to drug transport across the BBB and most importantly to put this in a timeframe that is fundamental for practical clinical translation.

In the treatment of ischemic stroke, for example, liposomes and other nanoparticles have shown great success in facilitating a wide range of pharmaceutical therapeutics to pass through the damaged BBB during the first few hours after reperfusion and act on the ischemic regions of the brain, reducing infarct sizes and helping to prevent further brain damage. The nanoparticles allow for higher circulation times and bioavailability of their encapsulated drugs to the brain tissues that would otherwise inefficiently crossed the BBB. However, these studies concentrated only on the very early stages after brain ischemia and, therefore, did not address the long-term pathological effects and the practicality of clinical translation. Neuroprotective drugs' main aim is to increase the therapeutic window compared to thrombolytic therapies. However, this was not the case in those studies as the focus was on intravenous injection around the reperfusion time or shortly after, which, limits their potential use to the first few hours after ischemic insult. A greater understanding of the BBB disruption and the timeframe of its breakdown in association with brain disorders is needed to fully achieve the potential of this approach. Furthermore, a critical factor in the clinical translation of this approach is the variability in the time-window of BBB disruption and recovery after different neurological conditions such as stroke and MS. This point is very critical for pharmacological treatments that require sustained drug exposure due to the limited window of BBB opening. Thus, in this case, the design of nanoparticle-based drug delivery with slow drug release over longer time would be more appropriate to ensure that the treatment can outlast the window of BBB opening. The residency time of the drug delivery system in the brain and mechanism of clearance are also very important parameters that are yet to be investigated. Moreover, care must be taken in the translation of preclinical findings into clinical setting due to several fundamental differences between the preclinical model brains compared to humans. Anatomical differences, size and volume variation are among many factors that could overestimate the therapeutic benefits of this approach.

There are several questions that still need to be answered. First, the mechanism by which those nanoparticles gain access through the damaged BBB and if this is different from one disorder to another or if those mechanisms change over time in the same disorder, and, second, if those nanoparticles able to pass the damaged BBB and reach the brain parenchyma, what their cellular fate and mechanism of drug release are. Third, the safety and impact of nanoparticles of on brain parenchyma is still an area that requires extensive investigation. The answers stay unclear at present and further work is required to understand these processes in more details.

One should also not dismiss the challenges that may accompany this approach. Although BBB disruption has been reported in several neurological disorders, the process of disruption is not homogeneous as regions of intact and disrupted BBB coexist. These observations were reported in clinical and preclinical studies and largely depend on the severity of the neurological condition. Based on that, inhomogeneous treatment may happen and could limit the therapeutic progress. Therefore, it is critical to understand the mechanisms behind BBB disruption for each neurological condition to be able to develop strategies to overcome these limitations.

\section{Funding}

This article was funded by the Division of Pharmacy and Optometry, University of Manchester.

\section{Declaration of Interest}

The authors have no relevant affiliations or financial involvement with any organization or entity with a financial interest in or financial conflict with the subject matter or materials discussed in the manuscript. This includes employment, consultancies, honoraria, stock ownership or options, expert testimony, grants or patents received or pending, or royalties. Peer reviewers on this manuscript have no relevant financial or other relationships to disclose.

\section{References}

Papers of special note have been highlighted as either of interest $(\cdot)$ or of considerable interest (••) to readers.

1. Davson H. Review lecture. The blood-brain barrier. J Physiol. 1976;255:1-28.

2. Reese TS, Karnovsky MJ. Fine structural localization of a blood-brain barrier to exogenous peroxidase. J Cell Biol. 1967;34:207-217.

3. Pincus JB, Kramer B. Comparative study of the concentration of various anions and cations in cerebrospinal fluid and serum. J Biol Chem. 1923;57:463-470. 
4. Kubie LS, Shults GM. Studies on the relationship of the chemical constituents of blood and cerebrospinal fluid. J Exp Med. 1925;11:685-686.

5. Banks WA. From blood-brain barrier to blood-brain interface: new opportunities for CNS drug delivery. Nat Rev Drug Discovery. 2016;15:275-292.

.- Very interesting review highlighting new opportunities for CNS drug delivery.

6. Liu S, Levine SR, Winn HR. Targeting ischemic penumbra Part II: selective drug delivery using liposome technologies. J Exp Stroke Transl Med. 2011;4:16-23.

7. Neuwelt EA, Bauer B, Fahlke C, et al. Engaging neuroscience to advance translational research in brain barrier biology. Nat Rev Neurosci. 2011;12:169-182.

8. Banks WA. Are the extracellular [correction of extracelluar] pathways a conduit for the delivery of therapeutics to the brain? Curr Pharm Des. 2004;10:1365-1370.

9. Levin VA. Relationship of octanol/water partition coefficient and molecular weight to rat brain capillary permeability. J Med Chem. 1980;23:682-684.

10. de Gregori S, de Gregori M, Ranzani GN, et al. Morphine metabolism, transport and brain disposition. Metab Brain Dis. 2012;27:1-5.

11. Au-Yeung SC, Rurak DW, Gruber N, et al. A pharmacokinetic study of diphenhydramine transport across the blood-brain barrier in adult sheep: potential involvement of a carrier-mediated mechanism. Drug Metab Dispos. 2006;34:955-960.

12. Sanchez-Covarrubias L, Slosky LM, Thompson BJ, et al. Transporters at CNS barrier sites: obstacles or opportunities for drug delivery? Curr Pharm Des. 2014;20:1422-1449.

13. del Amo EM, Urtti A, Yliperttula M. Pharmacokinetic role of L-type amino acid transporters LAT1 and LAT2. Eur J Pharm Sci. 2008;35:161-174.

14. Coker GT, Studelska D, Harmon S, et al. Analysis of tyrosine hydroxylase and insulin transcripts in human neuroendocrine tissues. Brain Res Mol Brain Res. 1990;8:93-98.

15. Skarlatos S, Yoshikawa T, Pardridge WM. Transport of [125I]transferrin through the rat blood-brain barrier. Brain Res. 1995;683:164171.

16. Zhang Y, Pardridge WM. Rapid transferrin efflux from brain to blood across the blood-brain barrier. J Neurochem. 2001;76:15971600.

17. Zhang Y, Pardridge WM. Mediated efflux of IgG molecules from brain to blood across the blood-brain barrier. J Neuroimmunol. 2001;114:168-172.

18. Vorbrodt AW, Dobrogowska DH, Ueno $M$, et al. Immunocytochemical studies of protamine-induced blood-brain barrier opening to endogenous albumin. Acta Neuropathol. 1995;89:491-499.

19. Herve F, Ghinea N, Scherrmann JM. CNS delivery via adsorptive transcytosis. Aaps J. 2008;10:455-472.

20. Kulkarni AD, Patel HM, Surana SJ, et al. Brain-blood ratio: implications in brain drug delivery. Expert Opin Drug Delivery. 2016;13:8592.

21. Hardy J, Selkoe DJ. The amyloid hypothesis of Alzheimer's disease: progress and problems on the road to therapeutics. Science. 2002;297:353-356.

22. Zlokovic BV. Clearing amyloid through the blood-brain barrier. J Neurochem. 2004;89:807-811.

23. Demeule $M$, Regina $A$, Jodoin J, et al. Drug transport to the brain: key roles for the efflux pump P-glycoprotein in the blood-brain barrier. Vascul Pharmacol. 2002;38:339-348.

24. Daneman R. The blood-brain barrier in health and disease. Ann Neurol. 2012;72:648-672.

25. Hellstrom M, Gerhardt $H$, Kalen M, et al. Lack of pericytes leads to endothelial hyperplasia and abnormal vascular morphogenesis. J Cell Biol. 2001;153:543-553.

26. Thomsen MS, Routhe LJ, Moos T. The vascular basement membrane in the healthy and pathological brain. J Cereb Blood Flow Metab. 2017;37:3300-3317.
27. Kacem K, Lacombe P, Seylaz J, et al. Structural organization of the perivascular astrocyte endfeet and their relationship with the endothelial glucose transporter: a confocal microscopy study. Glia. 1998;23:1-10.

28. Oberoi RK, Parrish KE, Sio TT, et al. Strategies to improve delivery of anticancer drugs across the blood-brain barrier to treat glioblastoma. Neuro Oncol. 2016;18:27-36.

29. Choonara YE, Kumar P, Modi G, et al. Improving drug delivery technology for treating neurodegenerative diseases. Expert Opin Drug Delivery. 2016;13:1029-1043.

30. Gross RE, Watts RL, Hauser RA, et al. Intrastriatal transplantation of microcarrier-bound human retinal pigment epithelial cells versus sham surgery in patients with advanced Parkinson's disease: a double-blind, randomised, controlled trial. Lancet Neurol. 2011;10:509-519.

31. Marks WJ Jr., Ostrem JL, Verhagen L, et al. Safety and tolerability of intraputaminal delivery of CERE-120 (adeno-associated virus serotype 2-neurturin) to patients with idiopathic Parkinson's disease: an open-label, phase I trial. Lancet Neurol. 2008;7:400-408.

32. Burgess A, Hynynen K. Noninvasive and targeted drug delivery to the brain using focused ultrasound. ACS Chem Neurosci. 2013;4:519-526.

33. Burgess KHA. Drug delivery across the blood-brain barrier using focused ultrasound. Expert Opin Drug Delivery. 2014;11:711-721.

34. Tamargo RJ, Rossell LA, Kossoff EH, et al. The intracerebral administration of phenytoin using controlled-release polymers reduces experimental seizures in rats. Epilepsy Res. 2002;48:145-155.

35. Pardridge WM. CSF, blood-brain barrier, and brain drug delivery. Expert Opin Drug Delivery. 2016;13:963-975.

.. Very interesting review by William M. Pardridge that draws the reader attention into very important misconceptions in the blood-CSF barrier and drug delivery such as drug distribution into CSF is not a measure of BBB permeability, and intrathecal injection of drug into the CSF distributes to blood and the surface of the brain, but not to more than 1-2 $\mathrm{mm}$ penetration depth into the brain parenchyma.

36. Tayebati SK, Nwankwo IE, Amenta F. Intranasal drug delivery to the central nervous system: present status and future outlook. Curr Pharm Des. 2013;19:510-526.

37. Pardridge WM. Drug transport across the blood-brain barrier. J Cereb Blood Flow Metab. 2012;32:1959-1972.

38. Joshi $S$, Ergin A, Wang $M$, et al. Inconsistent blood brain barrier disruption by intraarterial mannitol in rabbits: implications for chemotherapy. J Neurooncol. 2011;104:11-19.

39. Hynynen K, McDannold N, Vykhodtseva N, et al. Noninvasive MR imaging-guided focal opening of the blood-brain barrier in rabbits. Radiology. 2001;220:640-646.

40. Choi JJ, Wang S, Brown TR, et al. Noninvasive and transient bloodbrain barrier opening in the hippocampus of Alzheimer's double transgenic mice using focused ultrasound. Ultrason Imaging. 2008;30:189-200.

41. Mikitsh JL, Chacko AM. Pathways for small molecule delivery to the central nervous system across the blood-brain barrier. Perspect Medicin Chem. 2014;6:11-24.

42. Pardridge WM. The blood-brain barrier: bottleneck in brain drug development. NeuroRX. 2005;2:3-14.

.. An excellent review which provides an overview of the role of BBB on pharmaceutical development for CNT disorders.

43. Boado RJ, Hui EK, Lu JZ, et al. Drug targeting of erythropoietin across the primate blood-brain barrier with an IgG molecular Trojan horse. J Pharmacol Exp Ther. 2010;333:961-969.

44. Hornykiewicz O. L-DOPA: from a biologically inactive amino acid to a successful therapeutic agent. Amino Acids. 2002;23:65-70.

45. Pardridge WM, Oldendorf WH. Kinetic analysis of blood-brain barrier transport of amino acids. Biochim Biophys Acta, Biomembr. 1975;401:128-136.

46. Su TZ, Lunney E, Campbell G, et al. Transport of gabapentin, a gamma-amino acid drug, by system I alpha-amino acid transporters: a comparative study in astrocytes, synaptosomes, and $\mathrm{CHO}$ cells. J Neurochem. 1995;64:2125-2131. 
47. Ronaldson PT, Davis TP. Targeting blood-brain barrier changes during inflammatory pain: an opportunity for optimizing CNS drug delivery. Ther Deliv. 2011;2:1015-1041.

48. Lassmann H, Bruck W, Lucchinetti C. Heterogeneity of multiple sclerosis pathogenesis: implications for diagnosis and therapy. Trends Mol Med. 2001;7:115-121.

49. Bielekova B, Kadom N, Fisher E, et al. MRI as a marker for disease heterogeneity in multiple sclerosis. Neurology. 2005;65:10711076.

50. Leppert D, Lindberg RL, Kappos L, et al. Matrix metalloproteinases: multifunctional effectors of inflammation in multiple sclerosis and bacterial meningitis. Brain Res Brain Res Rev. 2001;36:249-257.

51. Moustafa RR, Baron JC. Clinical review: imaging in ischaemic stroke-implications for acute management. Crit Care. 2007;11:227.

52. Kochanek PM, Hallenbeck JM. Polymorphonuclear leukocytes and monocytes/macrophages in the pathogenesis of cerebral ischemia and stroke. Stroke. 1992;23:1367-1379.

53. Huang J, Upadhyay UM, Tamargo RJ. Inflammation in stroke and focal cerebral ischemia. Surg Neurol. 2006;66:232-245.

54. Zlokovic BV. Neurovascular mechanisms of Alzheimer's neurodegeneration. Trends Neurosci. 2005;28:202-208.

55. Ryu JK, McLarnon JG. A leaky blood-brain barrier, fibrinogen infiltration and microglial reactivity in inflamed Alzheimer's disease brain. J Cell Mol Med. 2009;13:2911-2925.

56. Prakash R, Carmichael ST. Blood-brain barrier breakdown and neovascularization processes after stroke and traumatic brain injury. Curr Opin Neurol. 2015;28:556-564.

57. van Vliet EA, da Costa Araujo S, Redeker S, et al. Blood-brain barrier leakage may lead to progression of temporal lobe epilepsy. Brain. 2007:130:521-534.

58. Aronica E, Gorter JA, Jansen GH, et al. Expression and cellular distribution of multidrug transporter proteins in two major causes of medically intractable epilepsy: focal cortical dysplasia and glioneuronal tumors. Neuroscience. 2003;118:417-429.

59. Shah GN, Price TO, Banks WA, et al. Pharmacological inhibition of mitochondrial carbonic anhydrases protects mouse cerebral pericytes from high glucose-induced oxidative stress and apoptosis. J Pharmacol Exp Ther. 2013;344:637-645.

60. McCaffrey G, Seelbach MJ, Staatz WD, et al. Occludin oligomeric assembly at tight junctions of the blood-brain barrier is disrupted by peripheral inflammatory hyperalgesia. J Neurochem. 2008;106:2395-2409.

61. Rosenberg GA. Neurological diseases in relation to the blood-brain barrier. J Cereb Blood Flow Metab. 2012;32:1139-1151.

62. Haley MJ, Lawrence CB.The blood-brain barrier after stroke: structural studies and the role of transcytotic vesicles.J Cereb Blood Flow Metab.2016;28:456-470.

- This study showed that BBB disruption significantly exacerbated in the presence of other comorbidities; however, the consequence of events contributing to BBB hyperpermeability has not significantly changed.

63. Knowland D, Arac A, Sekiguchi KJ, et al. Stepwise recruitment of transcellular and paracellular pathways underlies blood-brain barrier breakdown in stroke. Neuron. 2014;82:603-617.

- In this article, the authors developed a transgenic mouse strain in which endothelial tight junctions were labeled with eGFP. This allows dynamics changes of tight junction integrity to be monitored in vivo in real-time using multiphoton microscopy.

64. Drozdzik M, Bialecka M, Mysliwiec K, et al. Polymorphism in the P-glycoprotein drug transporter MDR1 gene: a possible link between environmental and genetic factors in Parkinson's disease. Pharmacogenetics. 2003;13:259-263.

65. Bartels AL, Willemsen AT, Kortekaas R, et al. Decreased blood-brain barrier P-glycoprotein function in the progression of Parkinson's disease, PSP and MSA. J Neural Transm. 2008;115:1001-1009.

66. McColl BW, Rothwell NJ, Allan SM. Systemic inflammation alters the kinetics of cerebrovascular tight junction disruption after experimental stroke in mice. J Neurosci. 2008;28:9451-9462.
67. Lorberboym M, Lampl Y, Sadeh M. Correlation of 99mTc-DTPA SPECT of the blood-brain barrier with neurologic outcome after acute stroke. J Nucl Med. 2003;44:1898-1904.

68. Brouns $R$, Wauters $A$, de Surgeloose $D$, et al. Biochemical markers for blood-brain barrier dysfunction in acute ischemic stroke correlate with evolution and outcome. Eur Neurol. 2011;65:23-31.

69. Kang EJ, Major S, Jorks D, et al. Blood-brain barrier opening to large molecules does not imply blood-brain barrier opening to small ions. Neurobiol Dis. 2013;52:204-218.

70. Minagar A, Alexander JS. Blood-brain barrier disruption in multiple sclerosis. Mult Scler. 2003;9:540-549.

71. Grossman Rl, Gonzalez-Scarano F, Atlas SW, et al. Multiple sclerosis: gadolinium enhancement in MR imaging. Radiology. 1986;161:721725.

72. Smith ME, Stone LA, Albert PS, et al. Clinical worsening in multiple sclerosis is associated with increased frequency and area of gadopentetate dimeglumine-enhancing magnetic resonance imaging lesions. Ann Neurol. 1993;33:480-489.

73. Ortiz GG, Pacheco-Moises FP, Macias-Islas MA, et al. Role of the blood-brain barrier in multiple sclerosis. Arch Med Res. 2014;45:687-697.

74. Baeten KM, Akassoglou K. Extracellular matrix and matrix receptors in blood-brain barrier formation and stroke. Dev Neurobiol. 2011;71:1018-1039.

75. Kwon I, Kim EH, del Zoppo GJ, et al. Ultrastructural and temporal changes of the microvascular basement membrane and astrocyte interface following focal cerebral ischemia. J Neurosci Res. 2009;87:668-676.

76. Nishioku T, Dohgu S, Takata F, et al. Detachment of brain pericytes from the basal lamina is involved in disruption of the blood-brain barrier caused by lipopolysaccharide-induced sepsis in mice. Cell Mol Neurobiol. 2009;29:309-316.

77. Nimmerjahn A, Kirchhoff F, Helmchen F. Resting microglial cells are highly dynamic surveillants of brain parenchyma in vivo. Science. 2005;308:1314-1318.

78. Kroll RA, Neuwelt EA. Outwitting the blood-brain barrier for therapeutic purposes: osmotic opening and other means. Neurosurgery. 1998;42:1083-1099.

79. Dasgupta A, Liu M, Ojha T, et al. Ultrasound-mediated drug delivery to the brain: principles, progress and prospects. Drug Discov Today Technol. 2016;20:41-48.

80. Cheng Z, Zhang J, Liu H, et al. Central nervous system penetration for small molecule therapeutic agents does not increase in multiple sclerosis- and Alzheimer's disease-related animal models despite reported blood-brain barrier disruption. Drug Metab Dispos. 2010;38:1355-1361.

.. This study showed that pathological BBB disruption in diseased models of Alzheimer's and multiple sclerosis resulted in little or no increase in permeability to small therapeutic molecules $(<1000 \mathrm{Da})$.

81. Torchilin VP. Multifunctional, stimuli-sensitive nanoparticulate systems for drug delivery. Nat Rev Drug Discovery. 2014;13:813-827.

82. Weissig V, Pettinger TK, Murdock N. Nanopharmaceuticals (part 1): products on the market. Int J Nanomed. 2014:9:4357-4373.

83. Tahover E, Patil YP, Gabizon AA. Emerging delivery systems to reduce doxorubicin cardiotoxicity and improve therapeutic index: focus on liposomes. Anticancer Drugs. 2015;26:241-258.

84. Al-Ahmady Z, Kostarelos K. Chemical components for the design of temperature-responsive vesicles as cancer therapeutics. Chem Rev. 2016;116:3883-3918.

85. Al-Ahmady ZS, Chaloin O, Kostarelos K. Monoclonal antibody-targeted, temperature-sensitive liposomes: in vivo tumor chemotherapeutics in combination with mild hyperthermia. J Control Release. 2014;196:332-343.

86. Ozbakir B, Crielaard BJ, Metselaar JM, et al. Liposomal corticosteroids for the treatment of inflammatory disorders and cancer. $J$ Control Release. 2014;190:624-636.

87. Crielaard BJ, Lammers T, Schiffelers RM, et al. Drug targeting systems for inflammatory disease: one for all, all for one. J Control Release. 2012;161:225-234. 
88. Allen TM, Cullis PR. Liposomal drug delivery systems: from concept to clinical applications. Adv Drug Delivery Rev. 2013;65:36-48.

- A nice review on liposomal drug delivery and clinical application.

89. Proffitt RT, Satorius A, Chiang SM, et al. Pharmacology and toxicology of a liposomal formulation of amphotericin B (AmBisome) in rodents. J Antimicrob Chemother. 1991;28:49-61.

90. Barenholz Y. Doxil(R)-the first FDA-approved nano-drug: lessons learned. J Control Release. 2012;160:117-134.

91. Maeda $\mathrm{H}$. The enhanced permeability and retention (EPR) effect in tumor vasculature: the key role of tumor-selective macromolecular drug targeting. Adv Enzyme Regul. 2001;41:189-207.

92. Wu NZ, Da D, Rudoll TL, et al. Increased microvascular permeability contributes to preferential accumulation of Stealth liposomes in tumor tissue. Cancer Res. 1993;53:3765-3770.

93. Hobbs SK, Monsky WL, Yuan F, et al. Regulation of transport pathways in tumor vessels: role of tumor type and microenvironment. Proc Natl Acad Sci U S A. 1998;95:4607-4612.

94. Huang SK, Martin FJ, Jay G, et al. Extravasation and transcytosis of liposomes in Kaposi's sarcoma-like dermal lesions of transgenic mice bearing the HIV tat gene. Am J Pathol. 1993;143:10-14.

95. Jiao $X, \mathrm{He} P, \mathrm{Li} Y$, et al. The role of circulating tight junction proteins in evaluating blood brain barrier disruption following intracranial hemorrhage. Dis Markers. 2015;860120:25.

96. Tzeng SY, Green JJ. Therapeutic nanomedicine for brain cancer. Ther Deliv. 2013;4:687-704.

97. Wei $X$, Chen $X$, Ying $M$, et al. Brain tumor-targeted drug delivery strategies. Acta Pharmaceutica Sinica B. 2014;4:193-201.

98. Adibhatla RM, Hatcher JF, Tureyen K. CDP-choline liposomes provide significant reduction in infarction over free CDP-choline in stroke. Brain Res. 2005;5:1-2.

99. Turjeman K, Bavli Y, Kizelsztein P, et al. Nano-drugs based on nano sterically stabilized liposomes for the treatment of inflammatory neurodegenerative diseases. PLoS One. 2015;10:e0130442.

100. Fukuta T, Ishii T, Asai T, et al. Treatment of stroke with liposomal neuroprotective agents under cerebral ischemia conditions. Eur J Pharm Biopharm. 2015;97:1-7.

101. Ishii T, Asai $T$, Oyama $D$, et al. Amelioration of cerebral ischemiareperfusion injury based on liposomal drug delivery system with asialo-erythropoietin. J Control Release. 2012;160:81-87.

102. Fukuta $T$, Ishii $T$, Asai $T$, et al. Real-time trafficking of PEGylated liposomes in the rodent focal brain ischemia analyzed by positron emission tomography. Artif Organs. 2014;38:662-666.

103. Cruz LJ, Stammes MA, Que I, et al. Effect of PLGA NP size on efficiency to target traumatic brain injury. J Control Release. 2016;223:31-41.

104. Fukuta T, Asai T, Sato A, et al. Neuroprotection against cerebral ischemia/reperfusion injury by intravenous administration of liposomal fasudil. Int J Pharm. 2016;506:129-137.

105. Kawaguchi AT, Haida $M$, Ohba $H$, et al. Liposome-encapsulated hemoglobin ameliorates ischemic stroke in nonhuman primates: longitudinal observation. Artif Organs. 2013;37:904-912.

106. Kaneda S, Ishizuka T, Sekiguchi A, et al. Efficacy of liposomeencapsulated hemoglobin in a rat model of cerebral ischemia. Artif Organs. 2014;38:650-655.

107. Hamadate N, Yamaguchi T, Sugawara A, et al. Liposome-encapsulated hemoglobin ameliorates impairment of fear memory and hippocampal dysfunction after cerebral ischemia in rats. J Pharmacol Sci. 2010;114:409-419.

108. Fukumoto D, Kawaguchi AT, Haida M, et al. Liposome-encapsulated hemoglobin reduces the size of cerebral infarction in rats: effect of oxygen affinity. Artif Organs. 2009;33:159-163.

109. Kawaguchi AT, Fukumoto D, Haida M, et al. Liposome-encapsulated hemoglobin reduces the size of cerebral infarction in the rat: evaluation with photochemically induced thrombosis of the middle cerebral artery. Stroke. 2007;38:1626-1632.

110. Kawaguchi AT, Yamano M, Haida M, et al. Effect of oxygen affinity of liposome-encapsulated hemoglobin on cerebral ischemia and reperfusion as detected by positron emission tomography in nonhuman primates. Artif Organs. 2017;41:336-345.
111. Campos-Martorell M, Cano-Sarabia M, Simats A, et al. Charge effect of a liposomal delivery system encapsulating simvastatin to treat experimental ischemic stroke in rats. Int J Nanomed. 2016;11:3035-3048.

112. Takanashi $Y$, Ishida T, Kirchmeier MJ, et al. Neuroprotection by intrathecal application of liposome-entrapped fasudil in a rat model of ischemia. Neurol Med Chir. 2001;41:107-113.

113. Liu $\mathrm{H}$, Jablonska A, Li Y, et al. Label-free CEST MRI detection of citicoline-liposome drug delivery in ischemic stroke. Theranostics. 2016;6:1588-1600.

114. Ramos-Cabrer P, Agulla J, Argibay B, et al. Serial MRI study of the enhanced therapeutic effects of liposome-encapsulated citicoline in cerebral ischemia. Int J Pharm. 2011;405:228-233.

115. Smith DA, Vaidya SS, Kopechek JA, et al. Ultrasound-triggered release of recombinant tissue-type plasminogen activator from echogenic liposomes. Ultrasound Med Biol. 2010;36:145-157.

116. Laing ST, Moody MR, Kim H, et al. Thrombolytic efficacy of tissue plasminogen activator-loaded echogenic liposomes in a rabbit thrombus model. Thromb Res. 2012;130:629-635.

117. Kandadai MA, Meunier JM, Hart K, et al. Plasmin-loaded echogenic liposomes for ultrasound-mediated thrombolysis. Transl Stroke Res. 2015;6:78-87.

118. Hoyte L, Kaur J, Buchan AM. Lost in translation: taking neuroprotection from animal models to clinical trials. Exp Neurol. 2004;188:200-204.

119. Wahlgren NG, Ahmed N. Neuroprotection in cerebral ischaemia: facts and fancies - the need for new approaches. Cerebrovasc Dis. 2004;17(suppl 1):153-166.

120. Sutherland BA, Minnerup J, Balami JS, et al. Neuroprotection for Ischaemic Stroke: translation from the bench to the bedside. Int $J$ Stroke. 2012;7:407-418.

121. Imaizumi S, Woolworth V, Fishman RA, et al. Liposome-entrapped superoxide dismutase reduces cerebral infarction in cerebral ischemia in rats. Stroke. 1990;21:1312-1317.

122. Phelan AM, Lange DG. Ischemia/reperfusion-induced changes in membrane fluidity characteristics of brain capillary endothelial cells and its prevention by liposomal-incorporated superoxide dismutase. Biochim Biophys Acta. 1991;5:97-102.

123. Stanimirovic DB, Markovic M, Micic DV, et al. Liposome-entrapped superoxide dismutase reduces ischemia/reperfusion 'oxidative stress' in gerbil brain. Neurochem Res. 1994;19:1473-1478.

124. Yun $X$, Maximov VD, Yu J, et al. Nanoparticles for targeted delivery of antioxidant enzymes to the brain after cerebral ischemia and reperfusion injury. J Cereb Blood Flow Metab. 2013;33:583-592.

125. Ghosh S, Das N, Mandal AK, et al. Mannosylated liposomal cytidine $5^{\prime}$ diphosphocholine prevent age related global moderate cerebral ischemia reperfusion induced mitochondrial cytochrome $c$ release in aged rat brain. Neurosci. 2010;171:1287-1299.

126. Fresta M, Puglisi G. Survival rate improvement in a rat ischemia model by long circulating liposomes containing cytidine-51-diphosphate choline. Life Sci. 1997;61:1227-1235.

127. Fresta M, Puglisi G. Biological effects of CDP-choline loaded long circulating liposomes on rat cerebral post-ischemic reperfusion. Int J Pharm. 1996;134:89-97.

128. Fresta M, Wehrli E, Puglisi G. Enhanced therapeutic effect of cytidine-5'-diphosphate choline when associated with GM1 containing small liposomes as demonstrated in a rat ischemia model. Pharm Res. 1995;12:1769-1774.

129. Fresta M, Puglisi G, Di Giacomo C, et al. Liposomes as in-vivo carriers for citicoline: effects on rat cerebral post-ischaemic reperfusion. J Pharm Pharmacol. 1994:46:974-981.

130. Adibhatla RM, Hatcher JF, Dempsey RJ. Citicoline: neuroprotective mechanisms in cerebral ischemia. J Neurochem. 2002;80:12-23.

131. Secades JJ, Alvarez-Sabin J, Castillo J, et al. Citicoline for acute ischemic stroke: a systematic review and formal meta-analysis of randomized, double-blind, and placebo-controlled trials. J Stroke Cerebrovasc Dis. 2016;25:1984-1996.

132. LeVine SM. Albumin and multiple sclerosis. BMC Neurol. 2016;16:0160564. 
133. Spencer JI, Bell JS, DeLuca GC. Vascular pathology in multiple sclerosis: reframing pathogenesis around the blood-brain barrier. J Neurol Neurosurg Psychiatry. 2017;31:2017-316011.

134. Ishii T, Asai T, Fukuta T, et al. A single injection of liposomal asialoerythropoietin improves motor function deficit caused by cerebral ischemia/reperfusion. Int J Pharm. 2012;439:269-274.

135. Agulla J, Brea D, Campos F, et al. In vivo theranostics at the peri-infarct region in cerebral ischemia. Theranostics. 2013;4: 90-105.

136. Omori N, Maruyama K, Jin G, et al. Targeting of post-ischemic cerebral endothelium in rat by liposomes bearing polyethylene glycol-coupled transferrin. Neurol Res. 2003;25:275-279.

137. Muldoon LL, Pagel MA, Kroll RA, et al. A physiological barrier distal to the anatomic blood-brain barrier in a model of transvascular delivery. AJNR Am J Neuroradiol. 1999;20:217-222.

138. Nance E, Timbie K, Miller GW, et al. Non-invasive delivery of stealth, brain-penetrating nanoparticles across the blood-brain barrier using MRI-guided focused ultrasound. J Control Release. 2014;189:123-132.

139. Åslund AKO, Berg S, Hak S, et al. Nanoparticle delivery to the brain - by focused ultrasound and self-assembled nanoparticle-stabilized microbubbles. J Control Release. 2015;220:287294.

140. Shen Y, Guo J, Chen G, et al. Delivery of liposomes with different sizes to mice brain after sonication by focused ultrasound in the presence of microbubbles. Ultrasound Med Biol. 2016;42:1499-1511.
141. Muldoon LL, Neuwelt EA. Dose-dependent neurotoxicity (seizures) due to deposition of gadolinium-based contrast agents in the central nervous system. Radiology. 2015 Dec;277(3):925-926.

142. Ose $A$, Kusuhara $H$, Endo $C$, et al. Functional characterization of mouse organic anion transporting peptide $1 \mathrm{a} 4$ in the uptake and efflux of drugs across the blood-brain barrier. Drug Metab Dispos. 2010;38:168-176.

143. Gao B, Hagenbuch B, Kullak-Ublick GA, et al. Organic anion-transporting polypeptides mediate transport of opioid peptides across blood-brain barrier. J Pharmacol Exp Ther. 2000;294:73-79.

144. Thompson BJ, Sanchez-Covarrubias L, Slosky LM, et al. Hypoxia/ reoxygenation stress signals an increase in organic anion transporting polypeptide 1a4 (Oatp1a4) at the blood-brain barrier: relevance to CNS drug delivery. J Cereb Blood Flow Metab. 2014;34:699-707.

145. Ronaldson PT, Finch JD, Demarco KM, et al. Inflammatory pain signals an increase in functional expression of organic anion transporting polypeptide $1 \mathrm{a} 4$ at the blood-brain barrier. J Pharmacol Exp Ther. 2011;336:827-839.

146. Doyle KP, Cekanaviciute E, Mamer LE, et al. TGFbeta signaling in the brain increases with aging and signals to astrocytes and innate immune cells in the weeks after stroke. J Neuroinflammation. 2010;7:1742-2094.

147. Thompson BJ, Ronaldson PT. Drug delivery to the ischemic brain. Adv Pharmacol. 2014;71:165-202.

148. State of the nation: stroke statistics. Stroke Association, UK, 2016. 